5. Ziakas PD, Zacharioudakis IM, Zervou FN, Grigoras C, Pliakos EE, Mylonakis E. Asymptomatic carriers of toxigenic C. difficile in longterm care facilities: a meta-analysis of prevalence and risk factors. PLoS One 2015;10:e0117195.

\section{Comparison of Rates of Drain-Related Ventriculitis According to Definitions Used}

To the Editor-We read with interest the recent comparison of ventricular drain-related ventriculitis (VDRV) according to which definitions were used. ${ }^{1}$ Reyes et $\mathrm{al}^{1}$ undertook a retrospective assessment of 52 cases of VDRV using 4 sets of definitions and found that using the National Healthcare Safety Network (NHSN) definitions resulted in substantially more cases of DDRV being identified.

We recently undertook a pilot study as a prelude to the introduction of a national surveillance system of VDRV, and as part of that process, we reviewed many definitions. ${ }^{2}$ However, because overreliance on culture can occur, we categorized VDRV as either definite or probable and allowed for the fact that bacteria isolated from the cerebrospinal fluid (CSF) could represent contamination (eg, a single sample with coagulase negative staphylococci). ${ }^{2}$ Of 45 cases of VDRV in 4 pilot centers, 20 of 28 cases categorized were definite.

Meningitis or ventriculitis following neurosurgery is complex, and the infection rate can be expressed as a percentage of patients with a drain inserted or preferably as the rate of infection per 1,000 catheter days. ${ }^{3-5}$ The latter metric better reflects the risk associated with device duration. Ramanan et $\mathrm{al}^{5}$ reviewed 35 studies, which included 752 infections, and found that the rate was lower for high-quality studies than for lower-quality studies. This effect contrasts with that found with most other infections, where better surveillance identifies higher rates of infection. This finding highlights the complexity of this area of study.

In a literature search of definitions used to diagnose VDRV, 16 unique definitions were retrieved. ${ }^{6}$ A positive CSF culture was required in $50 \%$ of these definitions, but no definitions mandated that more than 1 CSF culture be positive to confirm infection. However, only 7 of 16 definitions (44\%) were objective, that is, they relied on laboratory data and clinical findings that were not overly open to interpretation. ${ }^{6}$ This finding explains, in large part, the variation in infection rates described in the literature.

The decision to start antibiotics in a neurosurgical patient with a drain in situ is largely a clinical one and must be guided by the best interest of the individual patient. This often means that more patients with suspected ventriculitis or meningitis are empirically treated than are subsequently confirmed to have the infection. In a recent study from the Netherlands, 48 of 209 patients with suspected ventriculitis
(23\%) were started on empirical antibiotics after subarachnoid haemorrhage. ${ }^{7}$ However, in only 11 patients $(5 \%)$ were the CSF cultures positive. A high red blood cell count in the CSF, as might perhaps be expected in this group of patients, was statistically associated with CSF culture-negative cases. $^{7}$

While the greater availability of molecular methods to diagnose VDRV may assist in determining the microbial cause, there will always be a need to assess a combination of clinical features, microbiological results, and other CSF parameters such as a protein levels, glucose levels, and cell counts. Surveillance definitions, however, should allow for the complex nature of this condition and the difficulties in being certain of the diagnosis. Not all cases are clear cut, and a positive CSF culture does not always indicate VDRV, especially if the case involves an organism that may reside on the skin, hence, the higher rate of VDRV with NHSN definitions. ${ }^{1}$ Consequently, there is a need for international agreement on surveillance definitions that are practicable and as rigorous as possible. Establishing such definitions will facilitate comparisons between centers that can inform improvements in the care related to these invasive devices and, ultimately, in patient outcomes.

\section{ACKNOWLEDGMENTS}

Financial support: No financial support was provided relevant to this article.

Potential conflicts of interest: All authors report no conflicts of interest relevant to this article.

\section{Hilary Humphreys, MD, FRCPI, FRCPath; ${ }^{1,2}$ Peter J. Jenks, MD, MRCP, FRCPath; ${ }^{3}$ Jennie Wilson, $\mathrm{PhD}^{4}$}

\begin{abstract}
Affiliations: 1. Department of Clinical Microbiology, The Royal College of Surgeons in Ireland, Dublin, Ireland; 2. Department of Microbiology, Beaumont Hospital, Dublin, Ireland; 3. Department of Microbiology and Infection Prevention and Control, Derriford Hospital, Plymouth, United Kingdom; 4. Richard Wells Research Centre, University of West London, London, United Kingdom.

Address correspondence to Hilary Humphreys, MD, FRCPI, FRCPath, Department of Clinical Microbiology, RCSI Education and Research Centre, Beaumont Hospital, Dublin D09 YD60 (hhumphreys@rcsi.ie). Infect Control Hosp Epidemiol 2017;38:1268-1269

(C) 2017 by The Society for Healthcare Epidemiology of America. All rights reserved. 0899-823X/2017/3810-0026. DOI: 10.1017/ice.2017.175
\end{abstract}

\section{REFERENCES}

1. Reyes MM, Munigala S, Church EL, et al. Comparing external ventricular drains-related ventriculitis surveillance definitions. Infect Control Hosp Epidemiol 2017;38:574-579.

2. Humphreys H, Jenks P, Wilson J, et al. Surveillance of infection associated with external ventricular drains; proposed methodology and results from a pilot study. J Hosp Infect 2017;95:154-160.

3. van de Beek D, Drake JM, D, Tunkel AR. Nosocomial bacterial meningitis. N Eng J Med 2010;362:146-154. 
4. Humphreys H, Jenks PJ. Surveillance and management of ventriculitis following neurosurgery. J Hosp Infect 2015;13:52-58.

5. Ramanan M, Lipman J, Shorr A, Shankar A. A meta-analysis of ventriculostomy-associated cerebrospinal fluid infections. $B M C$ Infectious Dis 2015. doi: 10.1186/s12879-014-0712-z.

6. Lewis A, Wahlster S, Karinja S, Czeisler B, Kimberley WT, Lord AS. Ventriculostomy-related infections (VRI): the performance of different definitions for diagnosing infection. Br J Neurosurg 2016;30:49-56.

7. Hoogmoed J, van de Beek D, Coert BA, Horn J, Vandertop WP, Verbann D. Clinical and laboratory characteristics for the diagnosis of bacterial ventriculitis after aneurysmal subarachnoid hemorrhage. Neurocrit Care 2017; 26:362-370. 